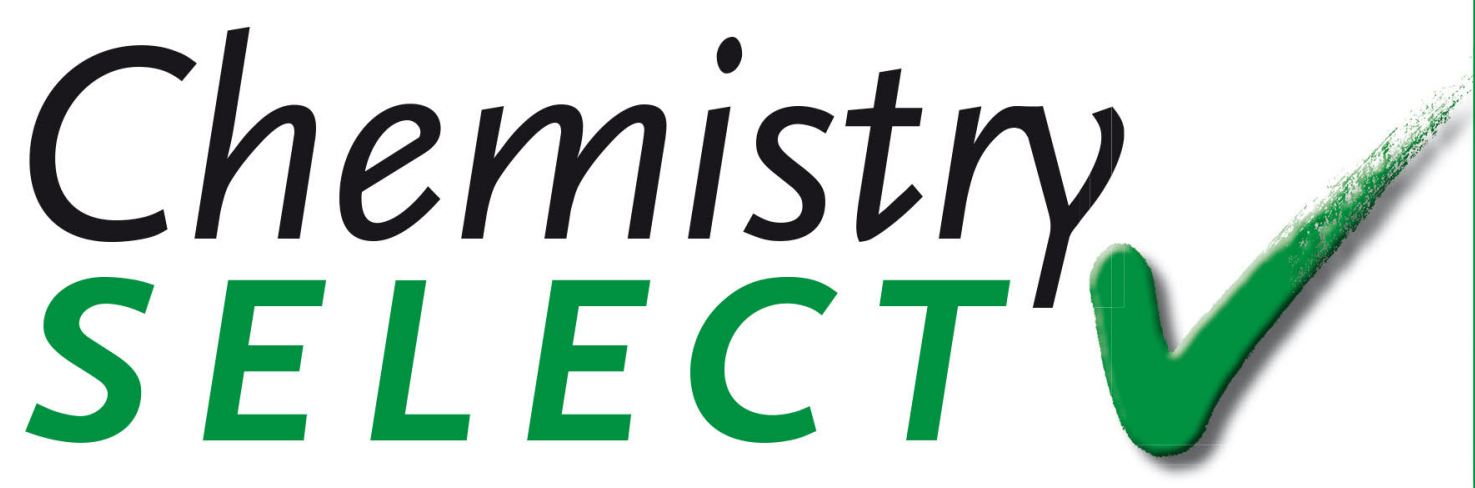

A journal of

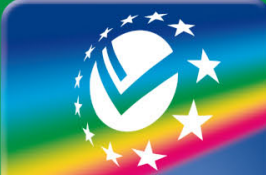

ChemPubSoc Europe

www.chemistryselect.org

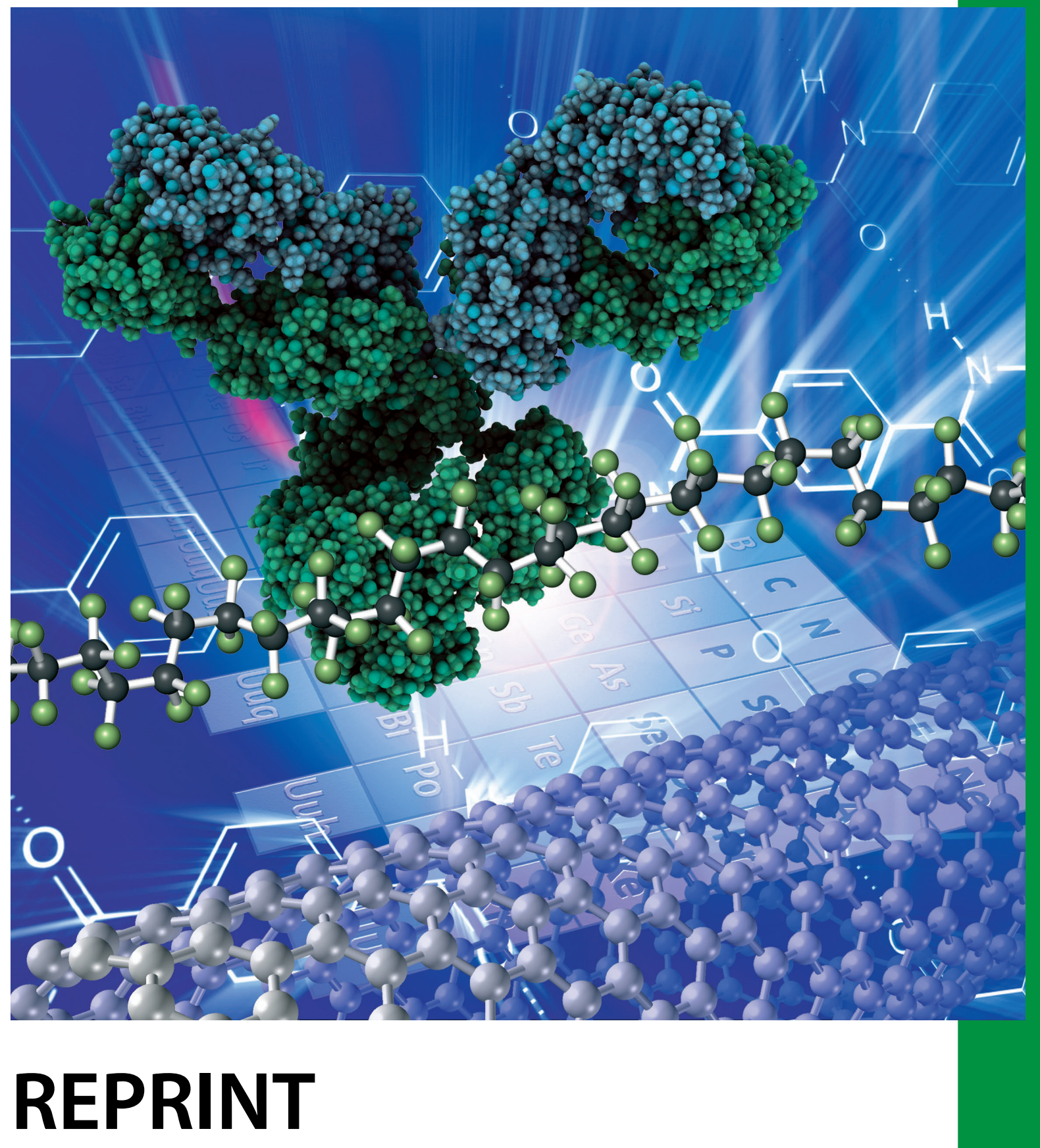

WILEY-VCH 


\title{
Reduction of Trichloromethyl to gem-Dichloromethyl Group with Triphenylphosphine and Water in Ethyl Acetate
}

\author{
Rafael Díaz-Hernández, ${ }^{[\mathrm{a}]}$ Ivann Zaragoza-Galicia, ${ }^{[\mathrm{a}]}$ Horacio F. Olivo, ${ }^{[\mathrm{b}]}$ and Moisés Romero- \\ Ortega*[a]
}

A novel and efficient way of reducing trichloromethyl to gemdichloromethyl compounds using environmentally friendly conditions has been developed. This reduction process consists of the treatment of a trichloromethyl compound with water and triphenylphosphine using ethyl acetate as solvent. This reaction proceeds in good yield when a strong electronwithdrawing group is attached to the trichloromethyl derivative.

\section{Introduction}

It is known that the gem-dichloromethyl group is an important backbone present in a number of biologically active compounds; they can act as inhibitors of cytochrome $\mathrm{P}-4500^{\prime} \mathrm{s}_{1}^{[1]}$ antibiotics, ${ }^{[2]}$ diuretics, ${ }^{[3]}$ and even some possess anti-cancer properties $^{[4]}$ see Figure 1. Furthermore, gem-dichloromethyl
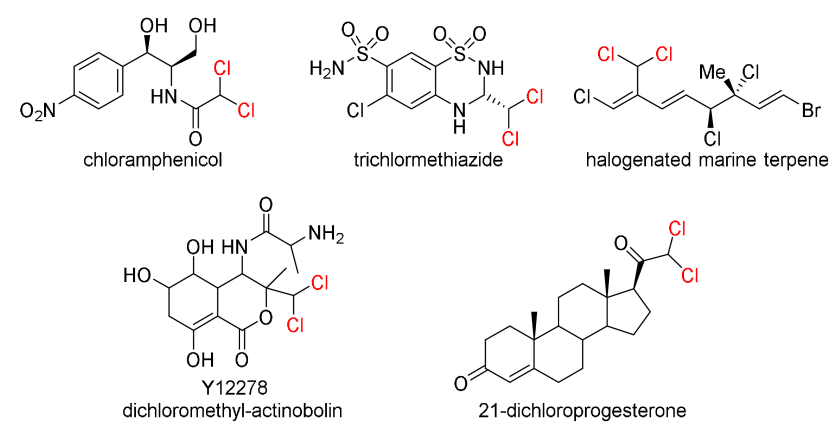

Figure 1. Biologically active gem-dichloromethyl compounds.

[a] R. Díaz-Hernández, Dr. I. Zaragoza-Galicia, Dr. M. Romero-Ortega Departamento de Química Orgánica, Facultad de Química Universidad Autónoma del Estado de México

Paseo Colon/Paseo Tollocan, Toluca, Estado de Mexico, Mexico E-mail: mromeroo@uaemex.mx

[b] Dr. H. F. Olivo

Medicinal and Natural Products Chemistry

The University of lowa lowa City, IA 52242, USA

Supporting information for this article is available on the WWW under https://doi.org/10.1002/slct.201702003 compounds are versatile intermediates for the synthesis of heterocycles, ${ }^{[5]} \alpha, \beta$-unsaturated ketones, ${ }^{[6]} \alpha$-chloroacrylates ${ }^{[7]}$ and cyclopropanes. ${ }^{[8]}$ Additionally, they are used as synthetic precursors to afford the corresponding aldehydes under basic conditions, ${ }^{[9]}$ as well as to generate free radicals that can add onto alkenes ${ }^{[10]}$ and alkynes. ${ }^{[11]}$

Traditional methods to prepare gem-dichloromethyl compounds involve chlorination of methyl ketones, which implicates using different types of chlorine sources, such as sulfuryl chloride, ${ }^{[12]}$ thionyl chloride, ${ }^{[13]}$ copper chloride ${ }_{,}^{[14]} \mathrm{N}$-chlorosuccinimide, ${ }^{[15]} \mathrm{N}$-chloro- $N$-methoxybenzenesulfonamide, ${ }^{[16]}$ trichloroisocyanuric acid, ${ }^{[17]}$ benzyltrimethylammonium tetrachloroiodate $^{[18]}$ and 1,3-dichloro-5,5-dimethylhydantoin (DCDMH). ${ }^{[19]}$ Although the transformation of the trichloromethyl group to the gem-dichloromethyl group by the mono-dechlorination (partial hydrogenation) is regarded as a direct and efficient method due to the easy preparation and the commercial availability of various trichloromethyl substrates, few methods have been described to allow the formation of gem-dichloromethyl compounds. Some of them require the use hydrogendonors, induced by transition metals, their complexes or salts at temperatures above $100^{\circ} \mathrm{C}_{1}^{[20]}$ a catalytic hydrogenation with $\mathrm{Pt} / \mathrm{C}_{1}^{[21]} n$-tributyltin hydride, ${ }^{[22]}$ sodium hydride, ${ }^{[23]}$ or Grignard reagents as electron donors to synthesize substituted $\alpha, \alpha$ dichloroketones. ${ }^{[24]}$ However, these methods display limitations and disadvantages such as poor yields, use of high temperatures, side-products formation, low chemoselectivity, and a limitation to tolerate different functional groups under reactions conditions. Therefore, the preparation of these compounds with a more efficient, simple and general route is highly desirable.

In 2011 Gilheany's group reported the use of 2-naphthol in toluene solution to the synthesis of pentachloroacetone ${ }^{[25]}$ in moderate yield from hexachloroacetone under the conditions of Appel reaction, ${ }^{[26]}$ however no systematic work was done using different substrates containing another functional group reactive. Recently, we reported a method to reduce trichloromethyl compounds to gem-dichloromethyl groups according to Appel's reaction protocol using methanol. ${ }^{[27]}$ Although this methodology affords excellent yields of gem-dichloromethyl derivatives with high chemoselectivity, the use of methanol produces as a by-product toxic residue (methyl chloride); this could be regarded as a disadvantage when large scale of the dichloromethyl compound is required. In this perspective, based in our previous experience, we decided to investigate a friendly protocol for the reduction of trichloromethyl com- 
pounds to gem-dichloromethyl using a green solvent and water as a source of protons.

\section{Results and Discussion}

We considered evaluating the reduction process of trichloromethyl compounds $\mathbf{1}$ in the presence of triphenylphosphine and water, employing tetrahydrofuran or ethyl acetate as a solvent, Scheme 1. Using trichloroacetophenone (1 a) as a

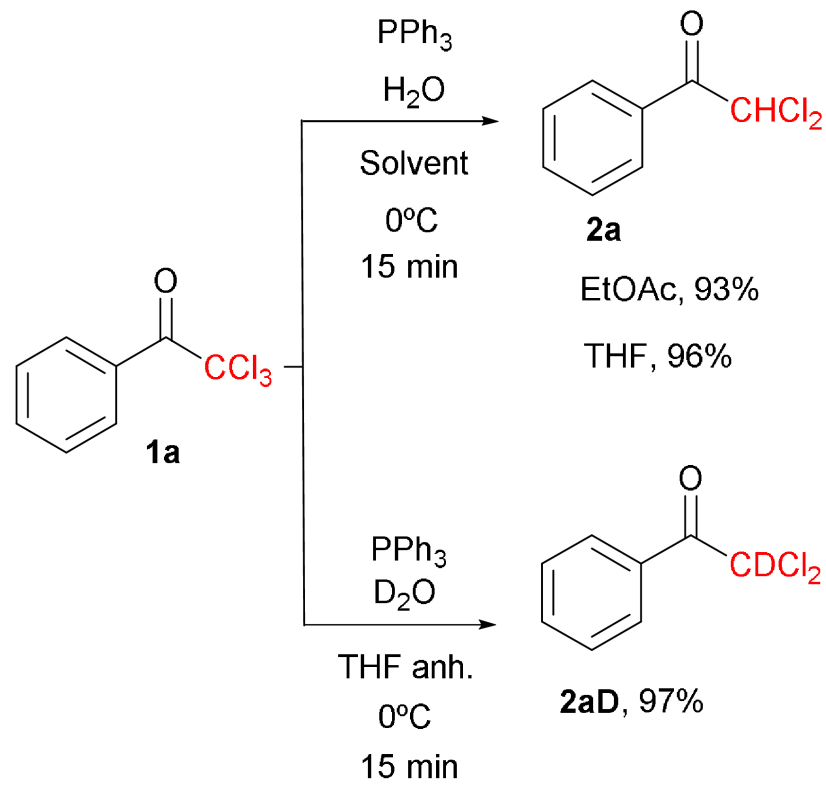

Scheme 1. Reduction of Trichroloacetophenone $\mathbf{1}$ a. Reaction conditions: $\mathbf{1}$ a (1.0 equiv), $\mathrm{PPh}_{3}$ (1.05 equiv), $\mathrm{H}_{2} \mathrm{O}$ or $\mathrm{D}_{2} \mathrm{O}$ (3.0 equiv), EtOAc or THF $(5.0 \mathrm{ml})$ at $0{ }^{\circ} \mathrm{C}$.

model, when it was reacted with 1.05 equiv. of triphenylphosphine and 2 equiv. of water in THF at room temperature, a mixture of $\mathbf{2}$ a along with monochloroacetophenone $(85 \%$ and $7 \%$ yield respectively) were obtained after $10 \mathrm{~min}$. When the reaction temperature was lowered to $0{ }^{\circ} \mathrm{C}$, dichloroacetophenone 2 a was obtained in $96 \%$ yield and the over-reduction product was not detected after $10 \mathrm{~min}$. We also investigated the influence of the amount of water in the reaction. Using three different amounts of water (1.0, 3.0 or 10.0 equiv) we observed no significant changes in the yield of the reduction reaction.

In order to verify that the water did in fact function as a source of protons, $\mathbf{1}$ a was reacted with 1.05 equiv. of triphenylphosphine, 3.0 equiv. of $\mathrm{D}_{2} \mathrm{O}$ in anhydrous THF, after $15 \mathrm{~min} 2 \mathrm{aD}$ was obtained in $97 \%$ yield. The presence of deuterium in $2 \mathrm{aD}$ was confirmed by ${ }^{1} \mathrm{HNMR}$ analysis, as we observed that the signal corresponding to the hydrogen of the dichloromethyl group $(6.70 \mathrm{ppm})$ showed a considerable decrease of intensity due to the presence of deuterium. Unfortunately, we only observe $55 \%$ of deuterium incorporation due possibly to the low quality of $\mathrm{D}_{2} \mathrm{O}$ used in this experiment (see the supporting information) verifying the source of protons (Scheme 1).

To make the reaction more attractive, 3.0 equiv. of water were reacted using ethyl acetate as the solvent, under these conditions 2 a was obtained after $15 \mathrm{~min}$ in 93\%. The experimental process is very simple to carry out and consist in the AcOEt elimination under reduced pressure (the AcOEt can be re-used) and obtain the respective product easily by flash chromatography. According to this result and given the high availability and the green solvent criteria of ethyl acetate, ${ }^{[28]}$ we decided to use these general conditions for the following experiments.

With this well-established protocol, we proceeded to extrapolate the methodology to different derivatives with similar reactivity to trichloroacetophenone $(\mathbf{1} \mathrm{a})$. The reductions of $\mathbf{1} \mathbf{b}$-h were carried out successfully obtaining the desired product in good yields (Table 1). Interestingly, when 4-methox-

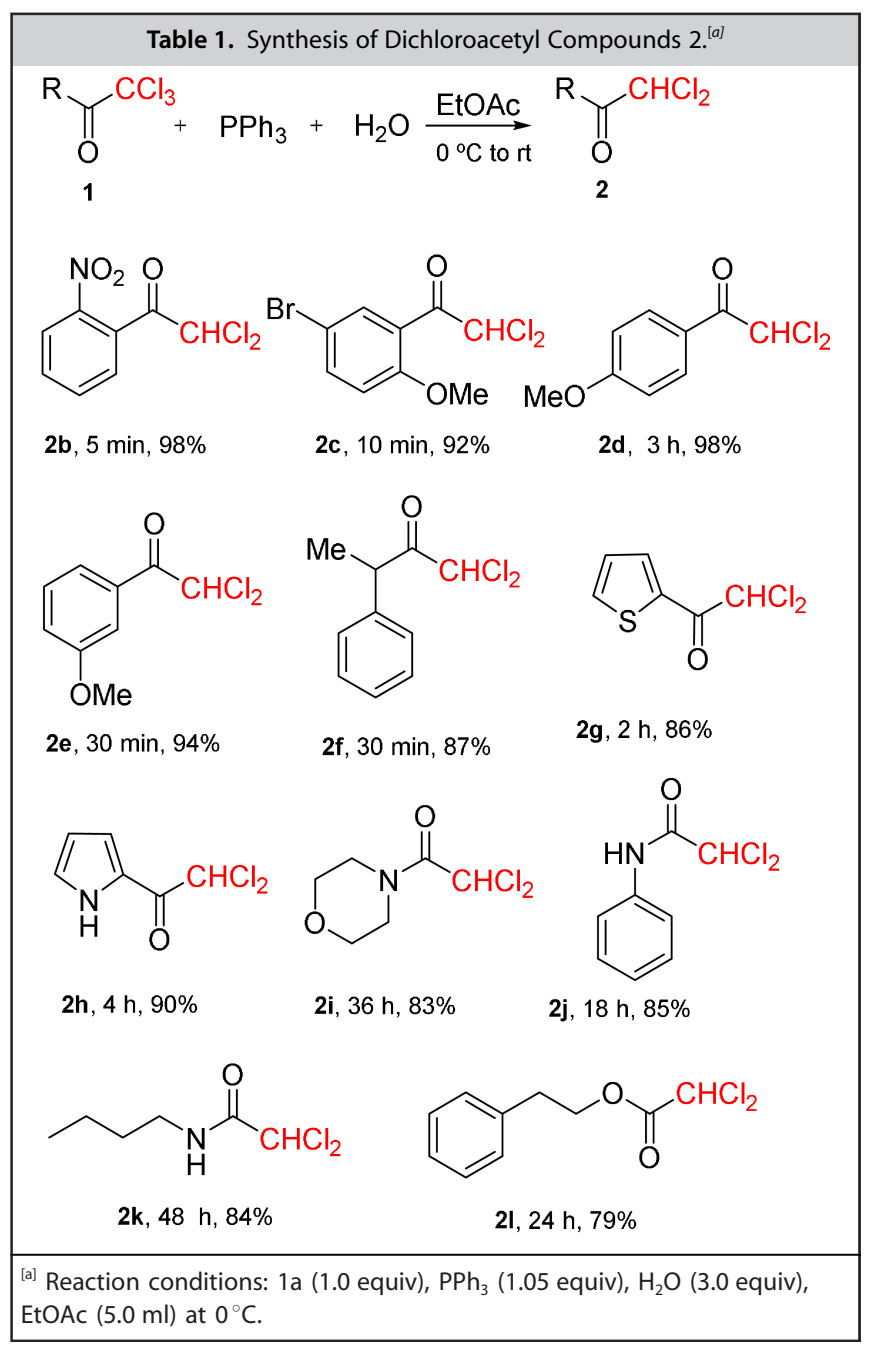

ytrichloroacetophenone (1 d), was treated under the same conditions, the reduction reaction time was $3 \mathrm{~h}$, a lot more time compared to the other trichloroacetyl derivatives ( $1 \mathbf{b}, \mathbf{1} \mathrm{c}$ and $1 \mathrm{e})$. A reason for this could be due to the electronic 
properties of the $p$-methoxy group which acts as an electrondonating group making the trichloroacetyl group less susceptible to reduction under these reaction conditions.

Additionally, the reductions of trichloroacetamides $\mathbf{1} \mathbf{i}-\mathbf{k}$ were carried out successfully to the desired dichloroacetyl compound $\mathbf{2} \mathbf{i}-\mathbf{k}$. The reaction times were longer as expected. This type of compounds are electron-rich in comparison with $\mathbf{1} \mathbf{b}$-e. Because of the presence of the unshared electron pair of nitrogen, this gives the trichloroacetyl group a richer electron density and in consequence longer reaction time. It is interesting to note that when the reaction was carried out in boiling ethyl acetate the reduction of $\mathbf{1} \mathbf{j}$ decreased (from 18 to $8 \mathrm{~h}$ ) and $\mathbf{2} \mathbf{j}$ was obtained in $\mathbf{8 7 \%}$ yield. This clearly establishes that the time of reduction of the trichloroacetyl group could be assumed to be generally shorter for others trichloromethyl compounds $\mathbf{2}$ and is not restricted merely to $\mathbf{2} \mathbf{j}$. Furthermore, the compounds $\mathbf{1} \mathbf{k}-\mathbf{l}$, were converted to the corresponding dichlorocompound $\mathbf{2} \mathbf{k}-\mathbf{I}$ without any problem. A library of compounds was created showing the compatibility of the reaction with different functional groups.

Finally, this reduction process was extrapolated to other trichoromethyl compounds possessing different scaffolds such as pyrimidines and some derivatives. As shown in Table 2, gem-

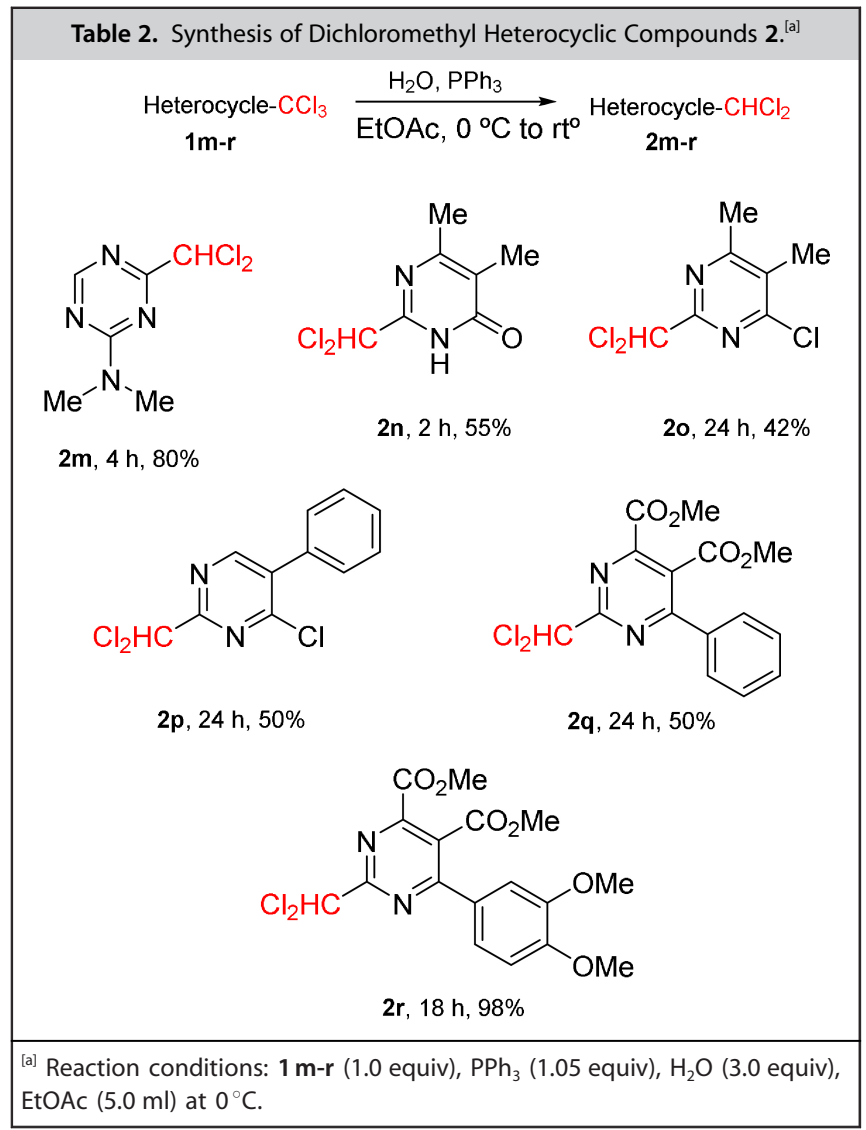

dichloromethyl $\mathbf{2} \mathbf{m}$ and $\mathbf{2 r}$ compounds were obtained in high yields under moderate periods of time (4 and $18 \mathrm{hrs}$ respectively). The reduction process was then tested to $\mathbf{1} \mathbf{n}-\mathbf{q}$, although thin layer chromatography analysis showed that the respective starting material was completely consumed and transformed to the dichloromethyl compound 2, low yields were obtained after column chromatography purification. We consider that this is probably due, in part, to the formation of a persistent insoluble white solid, which may arise from strong complexation of this kind of products with the triphenylphosphine oxide formed as by-product in this reduction reaction. For the cases of $\mathbf{1} \mathbf{o}$ and $\mathbf{1} \mathbf{p}$ we obtained exclusively the corresponding gem-dichloromethyl compound 2, without any dechlorination of the aromatic chloride. Notably, when the procedure was extended to the trichloromethyl derivatives $\mathbf{1} \mathbf{b}$ $1 \mathbf{p}$, the over-reduced products were not obverved. In fact, 1.0 equiv. of triphenyl phosphine is essential for the reaction to proceed even in excess of water.

We believe that this reduction process involves the formation of a key intermediate dichloromethyl carbanion A (Scheme 2). The nucleophilic phosphorus atom attacks the<smiles>[R]C(Cl)(Cl)[Si]CC[PbH2]</smiles>
1 A

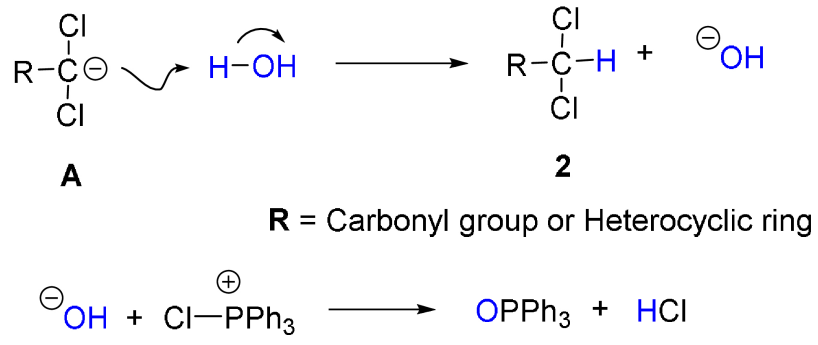

Scheme 2. Mechanism for the reduction of the trichloromethyl group to gem-dichloromethyl compound 2.

trichloromethyl compound, forming the stabilized carbanion A and chlorotriphenylphosphonium ion. Then, the high-energy intermediate $\mathbf{A}$, in the presence of water is protonated to give the respective gem-dichloromethyl compound $\mathbf{2}$ and one equivalent of hydroxyl ion, which reacts with chlorotriphenylphosphonium ion to give finally, triphenylphosphine oxide and hydrogen chloride. This mechanistic sequence is supported by the deuterium capture of the stabilized carbanion intermediate when the reduction process was carried out in $\mathrm{D}_{2} \mathrm{O}$, providing direct evidence on reaction mechanism. Additionally, we found a strong acidic media generated in the reaction media measured directly in the flask $(\mathrm{pH} \approx 1)$ when a large scale $(3.0 \mathrm{~g})$ reduction process of $1 \mathrm{a}$ was carried out in the presence of $1.0 \mathrm{~mL}$ of $\mathrm{H}_{2} \mathrm{O}$ in AcOEt solution, and 2 a was still isolated in high yield (93\%) after column chromatography. 


\section{Conclusions}

In summary, we have reported an efficient and simple process to prepare gem-dichloromethyl compounds from trichloromethyl derivatives using friendly environmental conditions. Under the optimal conditions only the gem-dichloromethyl compounds were obtained, and the monochloromethyl derivatives (over-reduction products) were not observed. Although, the efficiency of this method of reduction has been demonstrated by synthesizing a gram-scale the dichloromethyl derivative $\mathbf{2 a}$, this reaction may be applied to other trichloromethyl derivatives and is not restricted merely to trichloroacetophenone. This dechlorination reaction was highly chemoselective and is expected to be particularly useful in those instances where the trichloromethyl compounds are the usual precursors of gem-dichloromethyl derivatives, which are not affordable in good yields by other routes.

\section{Supporting Information Summary}

The supporting information provides complete experimental procedures and spectral data $\left({ }^{1} \mathrm{H},{ }^{13} \mathrm{C}\right.$ and HRMS) of new synthesized compounds $\mathbf{2} \mathbf{j}-\mathbf{1}, \mathbf{2} \mathbf{n}, \mathbf{2} \mathbf{~ o}, \mathbf{2 q}-\mathrm{r}$ and $\mathbf{2 a D}$.

\section{Acknowledgements}

The Consejo Nacional de Ciencia y Tecnologia is gratefully acknowledged for a postdoctoral fellowship to Ivann Zaragoza (CONACyT-231282) and grant (282953). The authors wish to thank M. Sc. Maria de las Nieves Zavala Segovia (CCIQS UNAM-UAEM) for obtaining NMR spectra, M. Sc. Lizbeth Triana Cruz for obtaining Mass spectra and to Prof. Joseph M. Muchowski (UNAM) for his interest in our work and helpful discussions.

\section{Conflict of Interest}

The authors declare no conflict of interest.

Keywords: Chemoselectivity - Dechlorination reaction - gemDichloromethyl compounds . Reduction process Trichloromethyl compounds

[1] J. R. Halpert, C. Balfour, N. E. Miller, L. S. Kaminsky, Molecular Pharmacology 1986, 30, 19-24.

[2] a) N. E. Miller, J. Halpert, Molecular Pharmacology 1986, 29, 391-398; b) F. Serra, P. Coutrot, M. Estève-Quelquejeu, P.Herson, T. K. Olszewski, C. Grison, Eur. J. Org. Chem. 2011, 1841-1847.

[3] R. Teraoka, Y. Matsushima, I. Sugimoto, K. Inoue, S. Morita, S. Kitagawa, Chem. Pharm. Bull. 2009, 57, 1343-1347.

[4] E. M. Antunes, A. F. Afolayan, M. T. Chiwakata, J. Fakee, M. G. Knott, C. E. Whibley, D. T. Hendricks, J. J. Bolton, D. R. Beukes, Phytochemistry 2011, $72,769-772$.
[5] a) G. A. Olah, K. K. Laali, Q. Wang, Prakash, G. K. S. Onium lons; JohnWiley \& Sons: New York, NY, 1998; b) K. Kim, J. Cho, S. C. Yoon, J. Chem. Soc, Perkin Trans. 1, 1995, 253-259.

[6] J. M. Concellón, H. Rodríguez-Solla, C. Concellón, P. Díaz, Synlett 2006, 6, 837-840.

[7] D. K. Barma, A. Kundu, H. Zhang, C. Mioskowski, J. R. Falck, J. Am. Chem. Soc. 2003, 125, 3218-3219.

[8] a) J. Fujiwara, A. Matsumura, Y. Matsuoka, J. Kiji, Bull. Chem. Soc. Jpn. 1976, 49, 829-831; b) N. Kawabata, T. Fuji, M. Naka, S. Yamashita, Bull. Chem. Soc. Jpn. 1977, 50, 1005-1015; c) C. Peppe, R. P. das Chagas, R. A. Burrow, J. Organomet. Chem. 2008, 693, 3441-3445.

[9] a) D. Bankston, Synthesis 2004, 2, 283-289; b) W. Li, J. Li, D. DeVincentis, T. S. Mansour, Tetrahedron Lett. 2004, 45, 1071-1074.

[10] D. Yang, Y.-L. Yan, B.-F. Zheng, Q. Gao, N.-Y. Zhu, Org. Lett. 2006, 8, 5757.

[11] A. J. Clark, G. M. Battlea, A. Bridg, Tetrahedron Lett. 2001, 42, 1999-2001.

[12] J. Prugh, A. Deana, J. Wiggins, Synthesis 1989, 554-556.

[13] C. Gabbutt, J. Hepworth, B. Heron, Tetrahedron 1994, 50, 5245-5254.

[14] J. A. Nobrega, S. M. C. Goncalves, C. Peppe, Synth. Commun. 2002, 32, 3711.

[15] a) I. Pravst, M. Zupan, S. Stavber, Tetrahedron 2008, 64, 5191-5199; b) D. Vrazic, M. Jereb, K. K. Laali, S. Stavber, Molecules 2013, 18, 74-96; c) K. Kedziora, F. R. Bisogno, I. Lavandera, V. Gotor-Fernandez, J. MontejoBernardo, S. Garcia-Granda, W. Kroutil, V. Gotor, ChemCatChem 2014, 6, 1066.

[16] X. Pu, Q. Li, Z. Lu, X. Yang, Eur. J. Org. Chem. 2016, 36, 5937-5940.

[17] G. A. Hiegel, K. B. Peyton, Synth. Commun. 1985, 15, 385-392.

[18] T. Kakinami, Y. Urabe, I. Hermawan, H. Yaminishi, T. Okamoto, S. Kajigaeshi, Bull. Chem. Soc. Jpn. 1992, 65, 2549-2551.

[19] a) Z. Chen, B. Zhou, H. Cai, W. Zhu, X. Zou, Green Chem. 2009, 11, 275278; b) Z. Zheng, B. Han, P. Cheng, J. Niu, A. Wang, Tetrahedron 2014, 70, 9814-9818.

[20] E. C. Chukovskaya, R. Kh. Freidlina, N. A. Kuz'mina, Synthesis 1983, 773784.

[21] a) T. Imanishi, Y. Fujiwara, Y. Sawama, Y. Monguchi, H. Sajiki, Adv. Synth. Catal, 2012, 354, 771-776; b) Y. Sawama, T. Imanishi, R. Nakatani, Y. Fujiwara, H. Sajiki, Tetrahedron 2014, 70, 4540-4546.

[22] a) P. S. Tiseni, R. Peters, Angew. Chem. Int. Ed. 2007, 46, 5325-5328; b) F. M. Koch, R. Peters, Angew. Chem. Int. Ed. 2007, 46, 2685-2689.

[23] N. G. Rivera, D. C. Becerril, C. Guadarrama-Perez, A. Covarrubias-Zuñiga, J. G. Avila-Zarraga, M. Romero-Ortega, Tetrahedron Lett. 2007, 48, 12011204.

[24] a) H. A. Essa, I. R. Lerrick, F. Tuna, W. R. Harrington, W. Clegg, M. J. Hall, Chem. Commun. 2013, 49, 2756-2758; b) H. A. Essa, I. R. Lerrick, E. Ciftci, W. R. Harrington, P.G. Waddell, W. Clegg, M. J. Hall, Organic \& Biomolecular Chemistry 2015, 13, 5793-5803.

[25] K. V. Rajendran, D. J. Carr, D. G. Gilheany, Tetrahedron Lett. 2011, 52, 7113-7115.

[26] R. Appel, R. Kleinstueck, K. D. Ziehn, Angew. Chem., Int. Ed. Engl. 1971, $10,132$.

[27] M. A. Romero-Reyes, I. Zaragoza-Galicia, H. F. Olivo, M. Romero-Ortega, J. Org. Chem. 2016, 81, 9515-9519.

[28] a) D. Prat, A. Wells, J. Hayler, H. Sneddon, C. R. McElroy, S. Abou-Shehada, P. J. Dunn, Green Chem. 2016, 18, 288-296; b) M. Y. Rios, E. Salazar, H. F. Olivo, Green Chem. 2007, 9, 459-462; c) E. G. Ankudey, H. F. Olivo, T. L. Peeples, Green Chem. 2006, 8, 923-926.

Submitted: August 30, 2017

Revised: October 19, 2017

Accepted: October 20, 2017 